\title{
Successful management of intralenticular Ozurdex injection causing cataract and intractable glaucoma
}

\author{
Kalpa Negiloni, Ronnie Jacob George, Sridharan Sudharshan, \\ Shweta Tripathi \\ Medical Research Foundation, Sankara Nethralaya, Chennai, Tamil Nadu, India
}

\begin{abstract}
A 50-year-old female presented with profound vision loss and was previously advised injection Ozurdex in the left eye. In the left eye, the anterior chamber was quiet, intraocular pressure (IOP) was $58 \mathrm{mmHg}$ and cataractous changes were noted with Ozurdex implant inside the lens substance. The left eye had glaucomatous cupping (0.9:1 CDR), bipolar rim thinning, inferior notch and healed choroiditis. The patient underwent phacoemulsification, trabeculectomy and mitomycin-C in the left eye. Visual acuity improved and IOP was under control. Although Ozurdex is effective, there are reports of complications related to the drug and implantation procedure. This case highlights an uncommon complication of an uncontrolled, persistent steroid response leading to glaucomatous optic atrophy and profound vision loss due to an accidental intralenticular implantation of Ozurdex. Our case reinforces the need for caution about the decision regarding the judicious use of intravitreal steroids and employment of appropriate technique.
\end{abstract}

Keywords: glaucoma, intravitreal corticosteroid implant, intralenticular Ozurdex, Ozurdex, posterior uveitis

\section{Introduction}

Intravitreal corticosteroid implants are widely available options for the treatment of macular oedema, non-infectious posterior uveitis, diabetic retinopathy, retinal venous occlusions and others. Ozurdex (Allergan, Inc., Irvine, CA, USA), the dexamethasone (DEX) drug delivery system, is a biodegradable intravitreal implant that delivers a sustained release of $700 \mu \mathrm{g}$ preservative-free DEX to the retina and vitreous. ${ }^{1,2}$ Although Ozurdex has been effective, there are reports of several complications related to the drug and the implantation procedure itself, such as its dislocation into the anterior chamber, accidental entry into the crystalline lens, increased intraocular pressure (IOP) and retinal detachment. We describe the successful management of a patient with increased IOP, advanced

Correspondence: Sridharan Sudharshan, 18, College Road, Nungambakkam, Chennai 600006, Tamil Nadu, India.

Phone: +91442727 1616

E-mail:drdharshan@gmail.com 
glaucomatous cupping and cataract due to accidental intralenticular injection of Ozurdex treated for geographic helicoid peripapillary choroiditis (GHPC). Our case highlights and reinforces the need for caution about the decision regarding the judicious use of intravitreal steroids and employment of appropriate technique.

\section{Case details}

A 50-year-old female presented to us with diminished vision in the right eye since 10 years and left eye in the last 2 months. She had been diagnosed with GHPC in both the eyes and had been administered injection Ozurdex in the left eye, 9 months ago. Her visual acuity recorded 2 months ago was 20/200 in the right eye and perception of light with inaccurate projection of rays in the left eye. The IOP was recorded as 11 and $38 \mathrm{mmHg}$ in the right and left eye, respectively. At presentation to us, her best-corrected visual acuity was 20/60; N10 at $30 \mathrm{~cm}$ in the right eye and hand movements close to face with relative afferent pupillary defect in the left eye. The slit-lamp examination of the right eye was unremarkable. The left-eye anterior chamber was quiet, with cataractous changes and Ozurdex implant inside the lens substance with a visible posterior capsule entry site (Fig. 1). Posterior capsule break was noted from 4 to 7 o'clock position at the area of entry of Ozurdex in the left eye. The anterior capsule was not breached. Lens opacification was noted along the injection track. Clinically, no definite leakage of lens material into vitreous cavity was noted. The IOP was 12 and $58 \mathrm{mmHg}$ in the right and left eye, respectively. The patient was on a combination of brimonidine and timolol twice daily and travoprost eye drops once daily in the left eye. She was on tablet acetazolamide thrice daily since a month. The gonioscopy revealed open angles in the right eye and closed angles in all quadrants which opened easily on compression with no Peripheral anterior synechiae in left eye. The retinal evaluation showed a healthy disc and healed choroiditis in the right eye. The left eye showed glaucomatous cupping with a 0.9:1 CDR, bipolar rim thinning, inferior notch and healed choroiditis. Investigations revealed raised ESR (32 $\mathrm{mm})$, normal chest X-ray and negative rapid plasma reagin, Mantoux and Treponema pallidum haemagglutination tests. The patient underwent a combined procedure of phacoemulsification with foldable intraocular lens implantation and trabeculectomy with mitomycin $C$ application under local anaesthesia. There were no intraoperative or post-operative complications. Post-operatively, the IOP was $6 \mathrm{mmHg}$ on first day and was maintained at $18 \mathrm{mmHg}$ at 1 week and thereafter. Her visual acuity improved to counting fingers at $3 \mathrm{~m}$. The post-operative period was uneventful. Topical steroids were tapered and stopped. 
Successful management of intralenticular Ozurdex injection causing cataract and intractable glaucoma
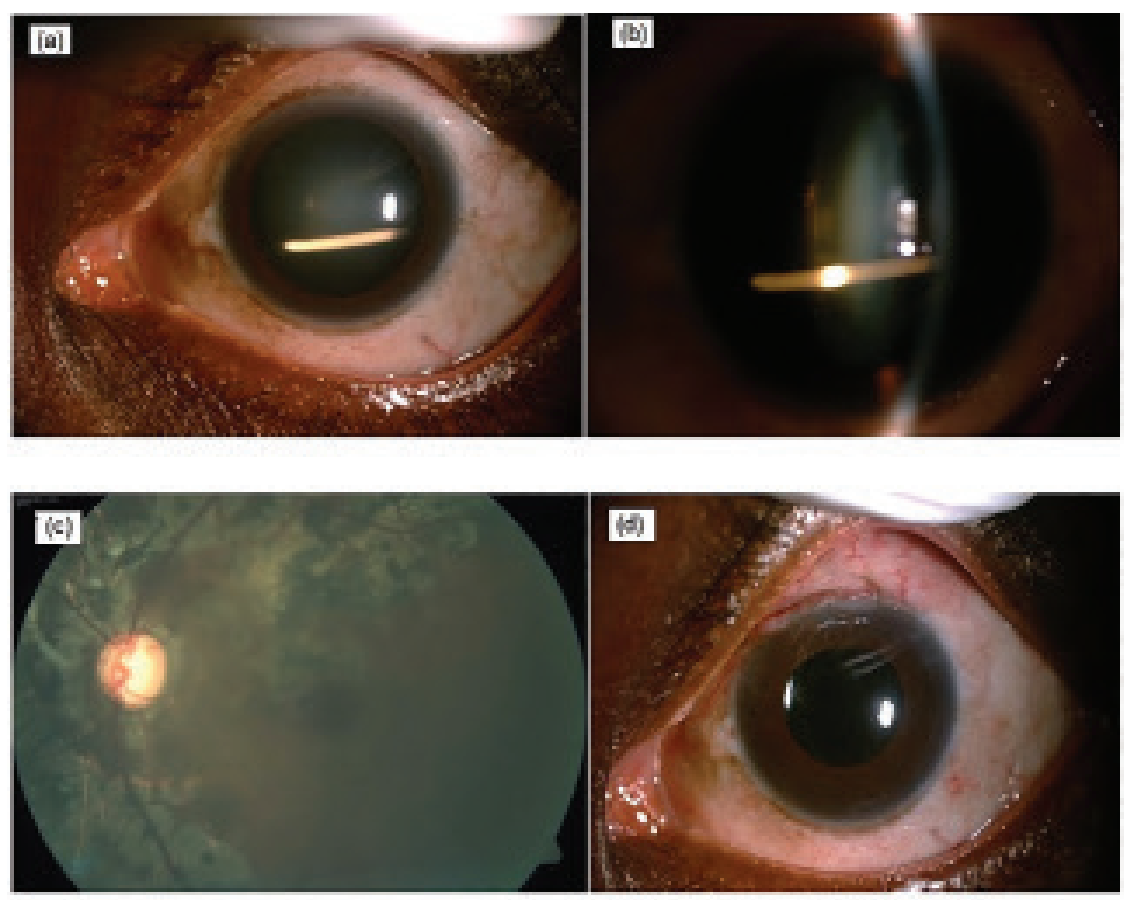

Fig. 1. Intralenticular Ozurdex implant in the left eye. (a and b) Intralenticular Ozurdex implant in the left eye. (c) Left eye fundus showed glaucomatous cupping, cup-disc ratio of 0.9 with bipolar rim thinning and patches of healed choroiditis. (d) Post-phacoemulsification with posterior chamber intraocular lens.

\section{Discussion}

Intravitreal corticosteroid implants have been used in the treatment of noninfectious intermediate, posterior and panuveitis with better safety profile. Commonly available intravitreal implants are biodegradable or non-biodegradable drug delivery systems such as Retisert, Iluvien and Ozurdex. These provide a sustained release of the drug to the posterior and intermediate segments of the eye. ${ }^{3,4}$

Ozurdex (Allergan, Inc, Irvine, CA, USA) DEX drug delivery system is a biodegradable intravitreal implant that delivers sustained release of $700 \mu \mathrm{g}$ preservative-free DEX to the retina and vitreous. ${ }^{1}$ The procedure is performed under controlled aseptic conditions and adequate anaesthesia to the periocular skin, eyelid and ocular surface. The tip of the needle of the Ozurdex injection is advanced within sclera for about $1 \mathrm{~mm}$ and redirected towards the centre of the eye until the vitreous cavity is entered. ${ }^{1}$ 
DEX implants have potential advantages of easy implantation, less frequent dosing, minimal side effects and biodegradability. There are anecdotal case reports of accidental entry of intravitreal implants to crystalline lens causing lens damage. The surgical intervention of cataract following intralenticular implantation has been managed variously by different authors. The decision of cataract surgery has depended on various factors such as the presence or absence of macular oedema and associated IOP (IOP). Surgery has been planned after resolution of macular oedema and till medical control of $\mathrm{IOP}^{5,6}$ or when vision drop was significant due to cataract. ${ }^{7}$

It is not always only the cataract that is the cause for vision loss but associated glaucoma too as was seen in our patient. In our case, Ozurdex was accidentally injected into the visual axis of the lens causing significant vision obstruction. Our patient also had a high IOP and was being managed medically elsewhere. The cause for profound vision loss in our patient was not only due to cataract but also due to glaucomatous changes of the disc secondary to raised persistent IOP.

In our patient, Ozurdex implant was injected accidentally into the visual axis of the lens causing profound vision loss due to cataract and intractable glaucoma. Our patient underwent phaco-trab as a treatment for both cataract and uncontrolled IOP. Our decision to plan the combined surgery was to prevent further irreversible loss due to glaucomatous optic nerve head damage.

In addition to cataractous change, our patient had severe intractable glaucoma when compared to other reported cases. ${ }^{8,9}$ This could possibly be due to the anterior location of the implant closer to the trabecular meshwork which is sensitive to the steroid. An exaggerated response in our case could also be partly related to extensive synechial angle closure. This significant high rise in IOP combined with delayed surgical treatment could have led to glaucomatous optic atrophy and severe vision loss.

In patients without other major complications such as cataract or glaucoma, options of repositioning or removal of implant alone have also been considered by some authors, but this can add to the further stress to PC causing a risk of external tear, anterior vitrectomy may be required, or implant can split or migrate to the anterior chamber. ${ }^{5}$ Intralenticular implantation could also be caused due to the use of larger diameter injectors that can cause considerable pressure on the globe and the pain experienced by the patient can provoke inadvertent eye or head movements. $7,8,10,11$

In our case, profound vision loss could be due to the presence of the implant in the visual axis, cataract or glaucomatous optic nerve head damage which needs early surgical intervention. In such cases, implant removal alone may not be sufficient. The interesting feature in our patient was that although Ozurdex was injected in our patient 9 months ago, the implant remained inside the lens without much 
decay. The pharmacokinetics and pharmacodynamics of a sustained-release DEX implant have been studied in monkeys, and the authors have concluded that the implant resulted in sustained levels of DEX and biological activity for 6 months, with the peak concentration of drug during the first 2 months. ${ }^{12}$ Although there is no definitive explanation for the long non-decay, in rare instances such as our cases the implant can remain like this inside the capsular bag for much longer time.

\section{Conclusion}

It is important to advise Ozurdex in select cases of non-infectious type of intermediate or posterior uveitis as adjuvant therapy. It is helpful in the treatment of macular oedema, especially in patients not tolerating systemic medications. Appropriate technique needs to be employed, and if there is an accidental injection into the, then our case indicates that it needs to be managed immediately, both for the complicated cataract and the associated high IOP, thus preventing irreversible vision loss due to glaucoma. Our case highlights an uncommon complication of an uncontrolled, persistent steroid response leading to glaucomatous optic atrophy and profound vision loss due to an accidental intralenticular implantation of Ozurdex. An exaggerated IOP response may be seen following intralenticular injection, and in such an event, early cataract surgery with or without additional filtration procedure and removal of the implant could prevent irreversible vision loss. Although GHPC resolved with treatment, there was aggravated IOP and cataract which was managed successfully surgically. Uveitis patients may already be at risk for secondary glaucoma aggravated by the use of steroids, and intravitreal steroids need to be used with caution. It is also important that the appropriate technique of implantation be followed to avoid such accidental injections which can result in irreversible visual complications.

\section{References}

1. Ozurdex" (Allergan, Inc, Irvine, CA, USA). http://www.ozurdex.com/. Last accessed 15 January 2018.

2. Haller JA, Kuppermann BD, Blumenkranz MS, et al. Dexamethasone DDS phase Il study group. Randomized controlled trial of an intravitreous dexamethasone drug delivery system in patients with diabetic macular edema. Arch Ophthalmol. 2010;128(3):289-296.

3. Myung JS, Aaker GD, Kiss S. Treatment of noninfectious posterior uveitis with dexamethasone intravitreal implant. Clin Ophthalmol. 2010;4:1423-1426.

4. Ghosn CR, Li Y, Orilla WC, et al. Treatment of experimental anterior and intermediate uveitis by a dexamethasone intravitreal implant. Invest Ophthalmol Vis Sci. 2011;52(6):2917-2923.

5. Sekeroglu MA, Anayol MA, Koc F, Tirhis H, Ozkan SS, Yilmazbas P. Intralenticular sustainedrelease dexamethasone implant: is it still effective on macular edema. Case Rep Ophthalmol. 2016;7(1):85-89.

6. Coca-Robinot J, Casco-Silva B, Armadß-Maresca F, García-Martínez J. Accidental injections of dexamethasone intravitreal implant (Ozurdex) into the crystalline lens. Eur J Ophthalmol. 2014;24:633-636. 
7. Fasce F, Battaglia Parodi M, Knutsson KA, et al. Accidental injection of dexamethasone intravitreal implant in the crystalline lens. Acta Ophthalmol. 2014;92:e330, e331.

8. Ram J, Agarwal AK, Gupta A, Gupta A. Phacoemulsification and intraocular lens implantation after inadvertent intracapsular injection of intravitreal dexamethasone implant. BMJ Case Rep. 2012;12:2012.

9. Sheen OS, Gepstein R, Assia El. Intralenticular Ozurdex implant: a case report. Clin Surg. 2017;2:1556.

10. Munteanu M, Rosca C. Repositioning and follow-up of intralenticular dexamethasone implant. J Cataract Refract Surg. 2013;39:1271-1274.

11. Roy R, Hegde S. Split Ozurdex implant: a caution. Can J Ophthalmol. 2013;48(1):e15, e16.

12. Chang-Lin JE, Attar M, Acheampong AA, et al. Pharmacokinetics and pharmacodynamics of a sustained-release dexamethasone intravitreal implant. Invest Ophthalmol Vis Sci. 2011;5;52(1):80-86. 